\title{
Development of the IT Convergence System for the Measurement of Dripping Speed of Medical Liquid using Difference Image
}

\author{
Jung-Sook Kim \\ Division of IT, Kimpo College, \\ Gimpo-si, Korea
}

\begin{abstract}
This paper describes the IT convergence system using a difference image, an erosion operation and a mobile application for the measurement of the dripping speed of the medical liquid in a drip chamber, which can estimate the remaining time using image processing from video frame information based on Android technology. The video frames were first made using a smartphone camera and we calculated the difference image between the $n$ image and the $(n+1)$ image and then changed into binary images using the threshold value. At this point, it is very important to find an optimal threshold value to recognize the dripping of the liquids. After that, we applied the erosion operation with threshold to detect the handshaking error of the smartphone camera. As a result, the user can adjust the dripping speed according to the doctor's prescription, exactly like watching the progress bar of a mobile application. The experiment results show that our method using image processing technique accurately measures the dripping speed for a wide range of speeds that are sufficient for ordinary practice.
\end{abstract}

Keywords: IT Convergence, Measurement of Dripping Speed of Medical Liquid, Difference Image, Threshold, Erosion Operation, Handshaking

\section{Introduction}

The phrase, 'IT convergence technology,' refers to the synergistic combination of conventional industry and IT, including advanced computing and communications. Applying cutting-edge technology to a traditional item equals a brand new product that is innovative and efficient [1]. For safety and efficacy in medical practice, there is usually a required $\mathrm{N}$ dripping speed. Newborns, adults, and people with cardiovascular or nephropathic conditions require different speeds. If the speed deviates too much from the prescribed value, there can be harmful effects. In extreme cases, this can even be life-threatening. Also, many drugs have required administration rates. If the speed is too low or too high, the intended effect cannot be achieved. In addition, during the normal dripping process, both nurses and patients must frequently check the speed and adjust it when it deviates. This practice takes up much of their time and attention. It is also difficult to estimate the remaining time for the completion of the dripping process, which makes scheduling a difficult task for nurses and the patients themselves. There are of course solutions to all these problems, such as the use of an infusion pump. However, the high price of infusion pumps (nowadays, usually over $\$ 1,000$ ) prevents their use in ordinary treatment, especially in developing countries. There are also devices that measure the dripping speed using infrared signals. An example is the intravenous drip feed monitor, which uses a combination of a diode and a phototransistor to detect drips. Another example is the apparatus for monitoring intravenous drips, which uses infrared or other types of emitters combined with a sensor to count the drips. The apparatus that counts

Copyright ( $) 2014$ Jung-Sook Kim. This is an open access article distributed under the Creative Commons Attribution License, which permits unrestricted use, distribution, and reproduction in any medium, provided the original work is properly cited. 
the number of drops can have mainly two purposes: first, to emit an alarm when the dripping speed deviates too much from the predetermined value; and second, to emit an alarm when the dripping has stopped to prevent infiltration $[2,3]$.

In this paper, we present these problems from a different perspective. We use a smartphone camera to record the dripping process and analyze its speed using the video and image processing techniques to measure the dripping speed of the liquids and to detect its end. The image processing has five phases to find the dripping occurred of the medical liquid. We first change the video and the video frames into binarized images using the threshold value. We also divide the frame into three parts and compare the difference of binary images between before the dripping binary image and after. At this point, it is very important to find the optimal threshold value to recognize the dripping of the medical liquid. And the middle areas of the divided frame are compared with the selected frame to no change frame for obtaining the threshold which can recognize the dripping before and after of the medical liquid. To remove the errors and handshaking the erosion operation is executed on the binary image. In addition, the user (or nurse) can adjust the dripping speed according to the doctor's prescription, exactly like watching the progress bar of a mobile application. Also, this system gives a user an alarm when the dripping speed deviates too much from a predetermined value. And it supports the function which indicates the focusing boundary to the drip chamber for taking a good frame using a smartphone camera. The experiment result showed that our method using video processing technique accurately measures the dripping speed for a wide range of speeds, which are sufficient for ordinary practice.

The structure of this paper is as follows. Section 2 briefly introduces the related works. Section 3 describes the development of the IT convergence system for the measurement of the dripping speed of the medical liquid using the video and image processing technique with a smartphone camera and section 4 presents its implementation details and the results. Finally, the conclusions are drawn and the future study is discussed in Section 5.

\section{Related Works}

There are some existing systems. One category of methods is to count the drops, and these are typically done by using optical sensors. Another method to count the drops is a touch method on the smartphone screen using smartphone application.

\subsection{Touch Method}

You can touch on the screen of smart phone to count the drops whenever the liquid begin to fall from drip chamber. After you touch the screen five times, the system displays the results and you can see the dripping speed and remaining time for the dripping process to finish. However this method is not always accurate to measure the dripping speed because the touch is done by hand.

\subsection{Monitoring by Video and Image Processing}

This work has two purposes: monitoring the dripping speed and alarming when the process finishes. Both functions can be performed by the same device, but uses different algorithms. In following illustrational embodiment, the device is made up chiefly of a containing box, a camera and processing unit. The drip chamber is placed at a fixed location inside the box so that its relative position to the camera is also fixed. For dripping speed monitoring we analyze a sequence of images. In each image, the same measurement is being computed, and requirement on this measurement is that it must display the same periodicity as the dripping itself. Such measurements from a sequence of images form a signal. To determine the 
dripping speed, discrete Fourier transform is performed and its largest non-constant component is recognized as the number of periods in the image sequence. Speed can then be computed accordingly. For detecting the end of the dripping process we find the location of the liquid surface in the drip chamber. However, since the dripping causes agitation in the liquid surface, we compute first the average of several consecutive images to eliminate the effect of this agitation. We then perform edge detection and identify the longest horizontal line segment inside the chamber as the liquid surface. Experiments show that our method provides accurate speed measurement for a wide range of actual speeds [2].

\section{Development of the Medical and IT Convergence}

\subsection{Recognition of the Dripping Speed Medical Liquid using Difference Image}

We developed a mobile application and a heuristic algorithm to recognize the difference binary images between before the dripping of the medical liquid and after the dripping of the medical liquid in a drip chamber from the frame information for the measurement of the dripping speed of the liquid. The nearly optimal threshold value was used to measure the dripping speed of the medical liquid using the image processing technique and the video that was obtained from a smartphone camera. The heuristic algorithm has five phases. At first, the user produced the video using a smartphone camera from the drip chamber. At this point, if smartphone camera does not support an auto-focus function, then the user had to keep the distance between the camera and the drip chamber at least $5 \mathrm{~cm}$. Had the user used a good camera, the range and distance of the camera would have been focused automatically. Secondly we calculated the difference image between the $n$ image and the $(n+1)$ image. The following numerical expression represents the difference image.

$$
D(x, y)=|I[n](x, y)-I[n+1](x, y)|
$$

Third then we calculated the threshold value using the iterative binarization method from the video frames. After that we changed the video frames into binarized images using the adjustable threshold value to distinguish the backgrounds from the objects. However, a drip chamber could be transparent or not. Also, the liquid could be transparent or not. As a result, finding an adjustable threshold value is very difficult. Moreover, we compared the obtained threshold values from each frame and selected the frames that changed the threshold value significantly or not according to the number of pixels. Then we divided the selected frame into three parts which are a top, a middle, and a bottom, and calculated the threshold value using iterative binarization. The middle areas of the divided frame are compared with the selected frame to no change frame for obtaining the threshold which can recognize the dripping before and after of the medical liquid. When dripping occurred, we can find two effects. One is that the number of light color from the binary image is increased compared to before dripping the medical liquid. The other is that the number of the dark color from the binary image is increased compared to before dripping the medical liquid. As a result, we decided the threshold value which is the number of light color or dark color. Using this threshold the binarization is executed to find white area. The white area from the binary image is the important factor which can distinguishes the dripping of the medical liquid. Forth the erosion operation is executed on the third phase result image to remove the handshaking and remaining errors. Finally the dripping is occurred when the number of white pixels is greater than the threshold value which is obtained threshold from third phase result. As a result, we found the point that had the greatest variation when dripping occurred in the best case, and we measured the dripping speed of the medical liquid and detected its end. Also, a 
user can control the dripping speed in a drip chamber exactly according to the doctor's prescription, in a manner similar to watching the progress bar of a mobile application.

\subsection{Erosion Operation for Avoiding the Handshaking}

Erosion is one of the two basic operators in the area of mathematical morphology, the other being dilation. It is typically applied to binary images, but there are versions that work on grayscale images. The basic effect of the operator on a binary image is to erode away the boundaries of regions of foreground pixels (i.e. white pixels, typically). Thus areas of foreground pixels shrink in size, and holes within those areas become larger [4]. We applied the erosion operation with threshold to detect the handshaking error of the smartphone camera. In the table 1, we can see the results of the erosion operation with threshold according to the background color to detect the handshaking error of the smartphone camera.

Table 1. Erosion Operation

\begin{tabular}{|c|c|c|}
\hline Background color & $\begin{array}{c}\text { The \# of erosion } \\
\text { operation }\end{array}$ & Threshold \\
\hline Black & 12 & 20 \\
\hline White & 16 & 10 \\
\hline
\end{tabular}

\section{Experiments and Results}

The medical and IT convergence system was implemented using the Java and C languages based on an Android environment [5, 6]. Figure 1-A and 1-B shows the results that were adjusted using the image processing technique. The threshold value was 128 for the image binarization from the original video frames that were obtained with a smartphone camera, the Galaxy S3 smartphone, which has a good resolution. We took frames for the experiment to detect the dripping in the drip chamber, and we took the variation that was greater than 100 . Figure 1-A and 1-B show the difference image between the image before the dripping and that after the dripping.

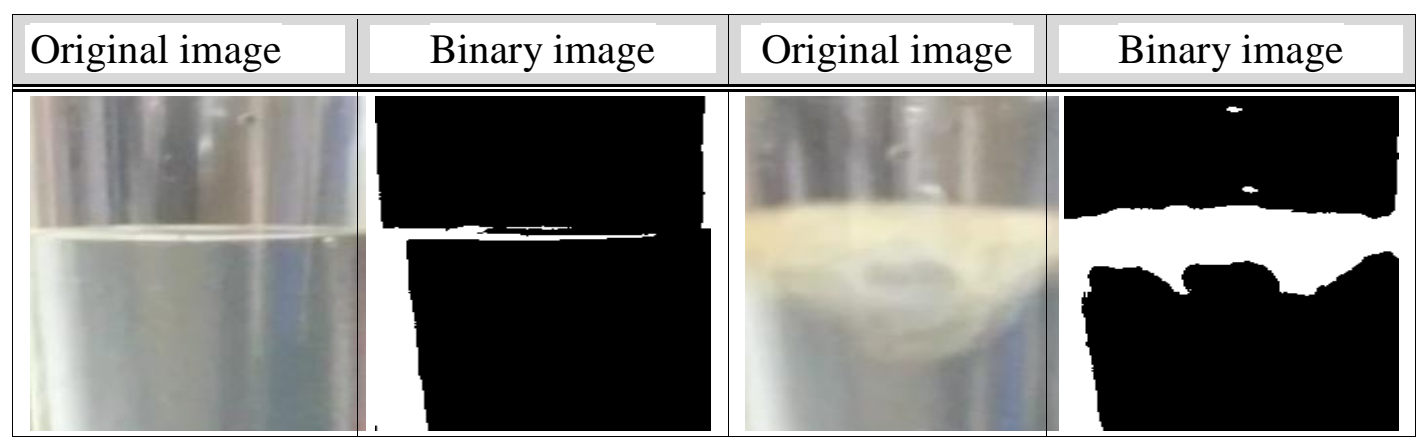

Figure 1-A. Before Dripping

Figure 1-B. After Dripping

The following figure 2-A, 2-B, 2-C, and 2-D show the recognition results which are applied the erosion operation. The $\mathrm{x}$ axis represents the number of frame and $\mathrm{y}$ is the result 
that is white area. The figure 2-A shows the result when erosion is executed on 4times and threshold value is 10 . The figure 2-B shows the result when erosion is executed on 10times and threshold value is 10 . The figure $2-\mathrm{C}$ shows the result when erosion is executed on 22 times and threshold value is 10 . The figure 2-D shows the best result when erosion is executed on 16times and threshold value is 10 . As the result, we selected that the number of near optimal erosion operation is 16times and near optimal threshold value is 10 .

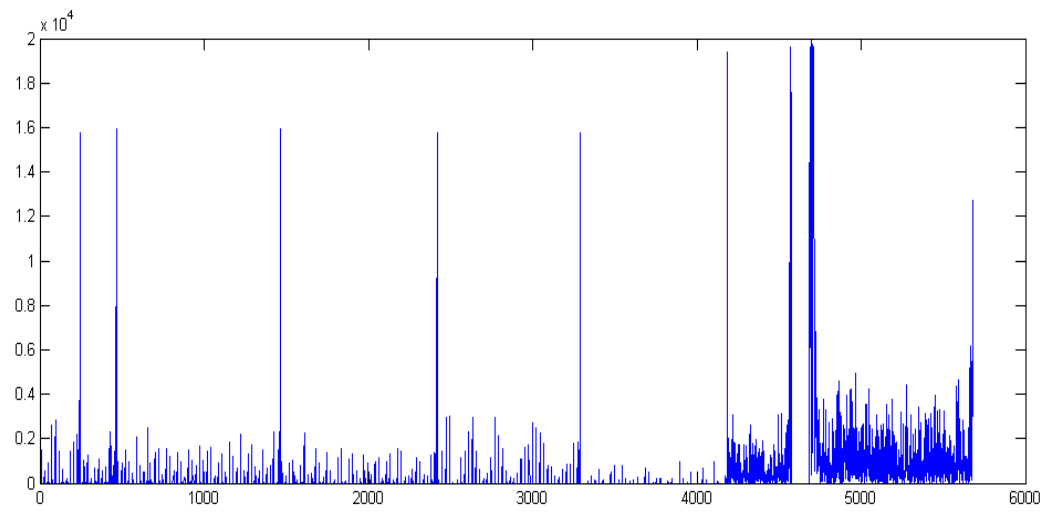

Figure 2-A. Histogram of Dripping Recognition of the Medical Liquid

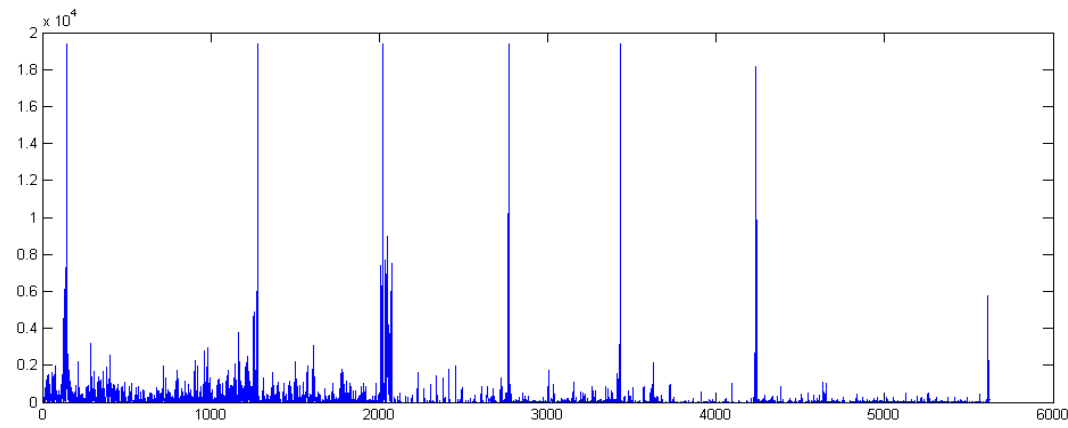

Figure 2-B. Histogram of Dripping Recognition of the Medical Liquid

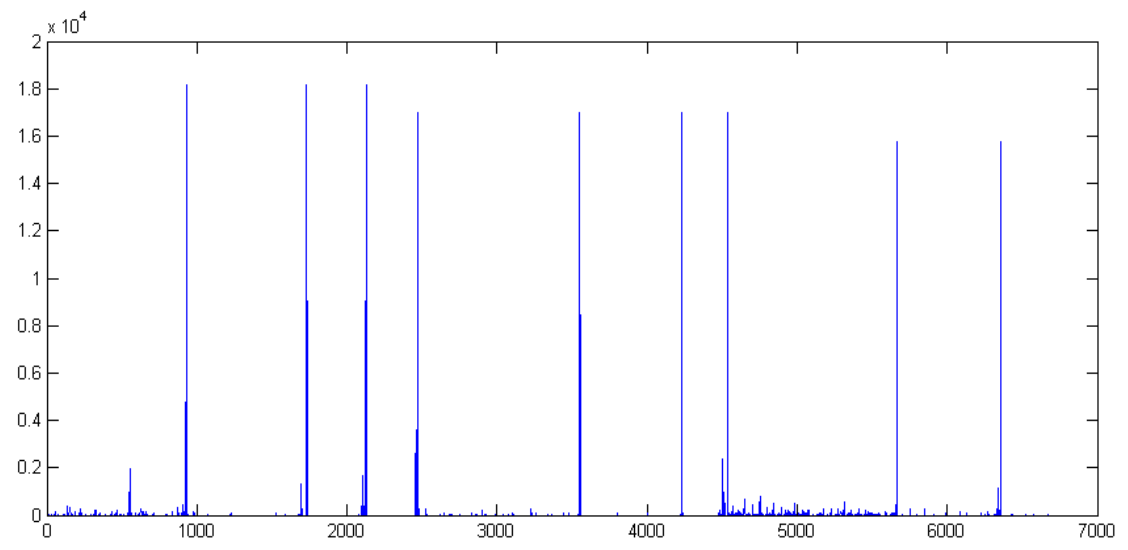

Figure 2-C. Histogram of Dripping Recognition of the Medical Liquid 


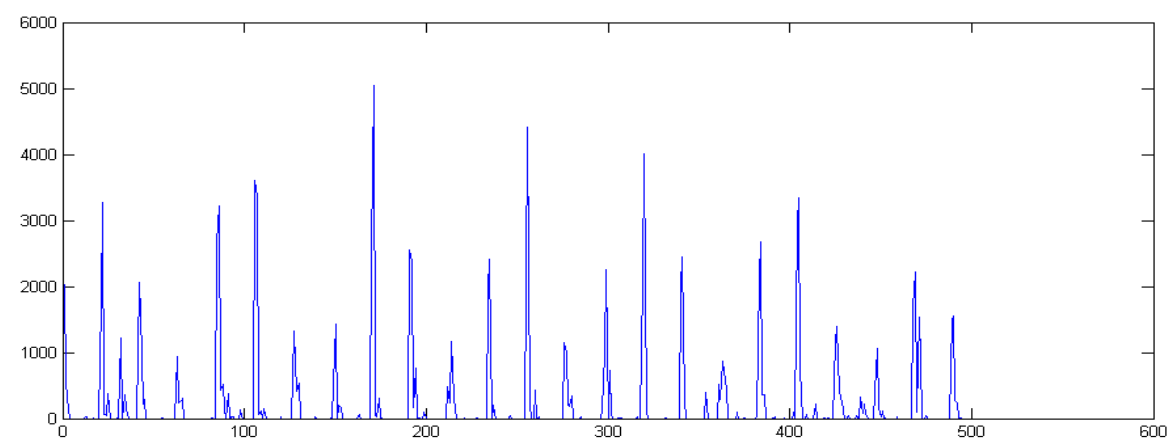

\section{Figure 2-D. The Best Result Histogram of Dripping Recognition of the Medical Liquid}

The parameters used to measure the dripping speed with the mobile application in real time, such as the number of drops, the amount of liquid in the drip chamber, the volume of the alarm, the number of measurements of the dripping speed of the liquid, and the near optimal threshold. Also, we can check a focusing boundary to the drip chamber in the checklist for presenting the guidelines to take the good frame, and we can check the checklist to avoid handshaking whether or not. The following figure 3 shows the estimated dripping speed of the liquid in real time using the mobile application on the left column of the first row, and the input dripping speed of the liquid, which was described in the doctor's prescription on the right column of the first row. As a result, we were able to precisely adjust the dripping speed according to the doctor's prescription by comparing the left column with the right column, which shows the dripping speed. Also, we can see the estimation drop intervals in real time using the mobile application on the left column of the second row; and on the right column of the second row, we can see the drop interval, which is the input according to the doctor's prescription as ordered by the user. In addition, the third row shows the progress status of the adjustment of the dripping speed using a color such as red or green in the table on top of the screen.

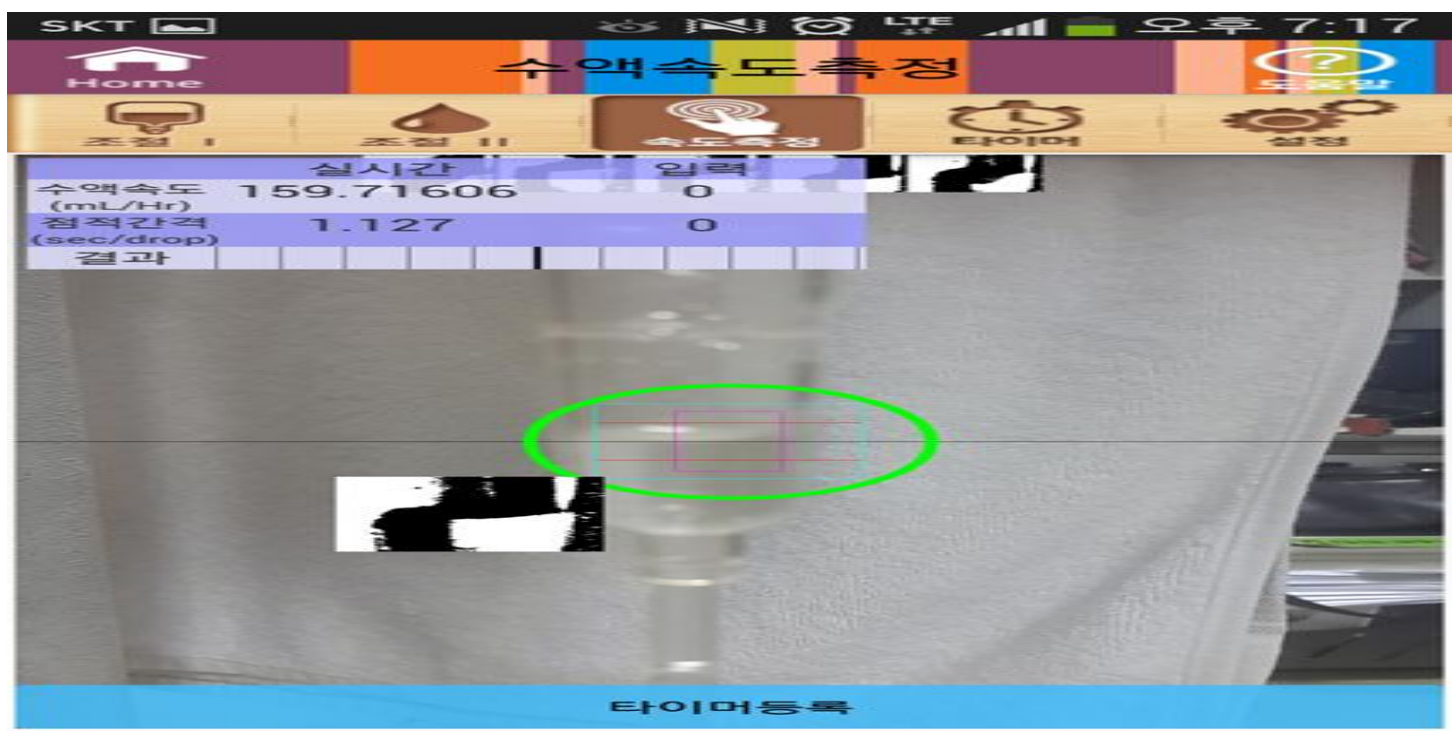

Figure 3. The Result of Dripping Speed on the Mobila Application 


\section{Conclusions and Future Work}

We developed the IT convergence system using a mobile application and a heuristic algorithm to recognize the states before and after the dripping of a liquid in a drip chamber from the video frame information for the measurement of the dripping speed of the liquid. We used a smartphone camera to record the dripping process and analyze its speed using the video and image processing techniques, to measure the dripping speed, and to detect its end. Moreover, we compared the obtained threshold values from each frame and selected the frames that significantly or only slightly changed the threshold value, the number of pixels. Then we divided the selected frame into three parts and calculated the threshold value using iterative binarization. As a result, we found the point that had the greatest variation when dripping occurred in the best case, and we measured the dripping speed of the liquid and detected its end. Also, a nurse can control the dripping speed in a medical drip chamber exactly according to the doctor's prescription, exactly like watching the progress bar of a mobile application. Also, this system gives a user an alarm when the dripping speed deviates too much from a predetermined value. And it supports the function which indicates the focusing boundary to the drip chamber for taking a good frame using a smartphone camera. The experiment result shows that our method using the image processing technique accurately measures the dripping speed for a wide range of speeds, which would suffice for ordinary practice. In the future study, we will use the proposed method to produce a production for sale.

\section{References}

[1] M. K. Kim, J. H. Park and Y. W. Cho, "Current Trends and Industrial Strategies of IT Convergence”, ETRI Journal, vol. 25, no. 1, (2010).

[2] United States patent application publication, "Monitoring by video and image processing", (2012) January.

[3] J. H. Kim, S. J. Wang, S. W. Lee, M. S. Kang, O. S. H. and K. C. You, "A report on the research on the accuracy of a flow regulator", Journal of the Korean Society of Emerg. Med., (2008), pp. 109-113.

[4] http://homepages.inf.ed.ac.uk/rbf/HIPR2/erode.htm.

[5] B. Bagewadi, C. Jatti, R. Koregaonkar, S. Somasagara, S. Chickerur and P. Mehta, "A parallel Video Frame Segmentation Approach on Multicore processors", international Journal of Control and Automation, vol. 7, no. 7, (2014), pp. 157-166.

[6] L. Ma, Y. Zhou, K. Liao, S. Liu, J. Qiao, Z. Han and J. Wang, "Design and Implementation of Controlling PC Wirelessly by Android Mobile Based on C/S Mode", International Journal of Control and Automation, vol. 7, no. 7, (2014), pp. 157-166. 
International Journal of Bio-Science and Bio-Technology Vol.7, No.1 (2015), 